\title{
Micromechanical modeling of random or imperfect composites
}

\author{
M. Šejnoha \& J. Zeman \\ Czech Technical University in Prague, Faculty of Civil Engineering, \\ Department of Structural Mechanics, Prague, Czech Republic
}

\begin{abstract}
A class of heterogeneous material systems often regarded as random or imperfect composites is addressed in this paper. The literature now offers a number of contributions that open the way to the analysis of large material systems with complex microstructures while taking advantage of popular micromechanics based approaches building on periodicity and first order homogenization techniques. Until recently the attention has been mostly limited to rather classical material systems such as unidirectional fibrous composites and textiles with emphasis on various types of imperfections developed during fabrication process on both microscale (level of fiber bundles) and meso-scale (level of textile geometry). From the basic mechanics point of view, however, it appears logical to exploit the essential principles of the proposed procedures in bridging the gap between mechanical and civil engineering applications. In this regard, historical masonry structures classified as systems composed of more than one material component serve as a typical example of civil engineering applications, which may benefit from standard first order homogenization schemes extended to account for possibly irregular arrangement of individual stone blocks. In this contribution, both groups of material systems will be treated on the same footing demonstrating the applicability of basic homogenization techniques as well as similarities between various heterogeneous material systems when referred to as random or imperfect.

Keywords: random and imperfect composites, textiles, masonry, periodic unit cell, two-point probability function.
\end{abstract}




\section{Introduction}

Techniques of numerical first-order homogenization have acquired a considerable attention particularly in applications where detailed numerical analysis of complex heterogeneous material systems proved to be prohibitively expensive. Natural assumption of the existence of periodic microstructure, e.g., periodic distribution of fibers in the metal matrix or ceramic matrix composites [1], often serve as the point of departure. The resulting homogenized or effective material parameters are then used in large scale structural analysis. Unfortunately, in the vast majority of real material systems the assumption of periodicity generally fails owing to the presence of various types of geometrical imperfections usually developed during fabrication. Giving up the benefit of periodic fields in such cases seems, therefore, reasonable but definitely not very practical. Instead, the recently introduced concept of statistically equivalent periodic unit cell (SEPUC) [2, 3, 4], appears as a suitable method of attack.

The leading idea of this approach is to replace a complex non-periodic microstructure by a certain periodic unit cell (PUC), which still optimally resembles the original microstructure in a proper sense. Here, this objective is formalized as a difference between appropriate statistical descriptors related to the original media and the periodic unit cell, respectively. If the original microstructure and the periodic unit cell is described by an identical set of parameters, this problem coincides with reconstruction of random materials. If the periodic unit cell is described by a substantially smaller number of parameters in order to reduce the problem complexity, it leads to a problem of the best approximation within the selected statistical descriptors.

It will be shown in subsequent paragraphs that the proposed technique is applicable not only to more or less classical types of composites represented here by textiles, but also to more conventional class of material systems such as historical masonry structures.

The principle idea is evident from Figs. 1-3 showing images of real material or structural systems with corresponding simplified representative volume elements (RVE) presented in terms of certain statistically equivalent periodic unit cells. When referring to textiles, Figs. 1 and 2, the crucial sources of imperfections are attributed to generally random arrangement of fibers within the bundle crosssection (microlevel) and the waviness, misalignment and/or non-uniform crosssectional aspect ratio of individual bundles in the longitudinal direction (mesolevel). Qualitatively similar types of imperfections associated with irregular arrangement of stone blocks, both in terms of block sizes and their location, together with a variable thickness of the mortar phase are often encountered when dealing with historical masonry structures. A typical example is plotted in Fig. 3(a) showing a parapet wall of the Charles Bridge in Prague.

The stepping stone in the analysis of all systems is the possibility to replace the original color images, Figs. 1(a)-3(a), by their binary counterparts, Figs. 1(b)3(b). The latter representatives of the true micro or meso-structures are further exploited in the next section when deriving various statistical descriptors. Match- 


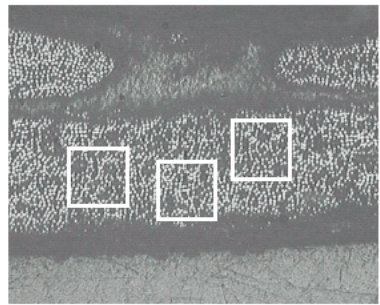

(a)

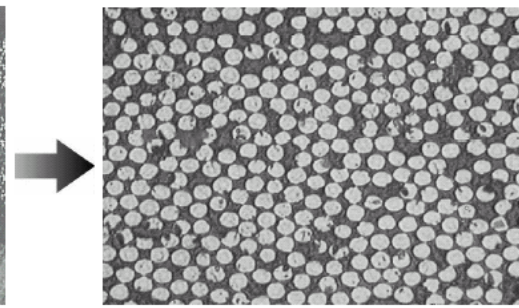

(b)

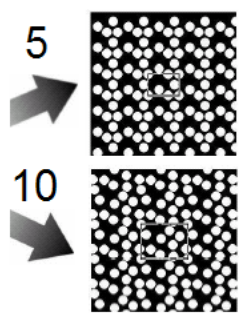

(c)

Figure 1: a) Micrograph of random fibrous composite, b) Binary image, c) 5-fiber and 10-fiber SEPUC.

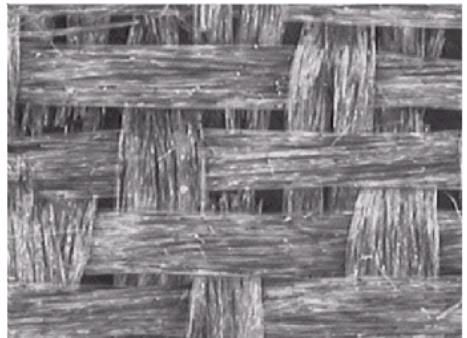

(a)

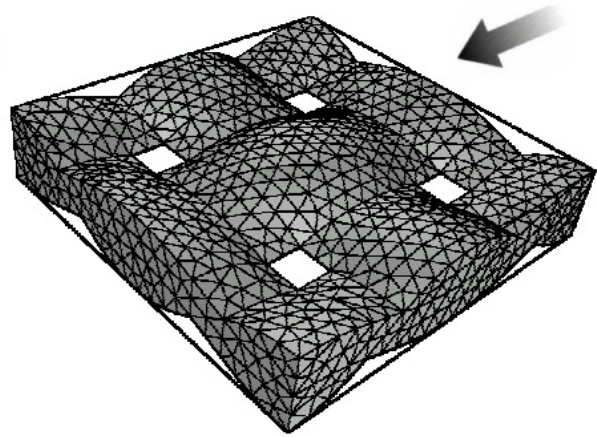

(c)

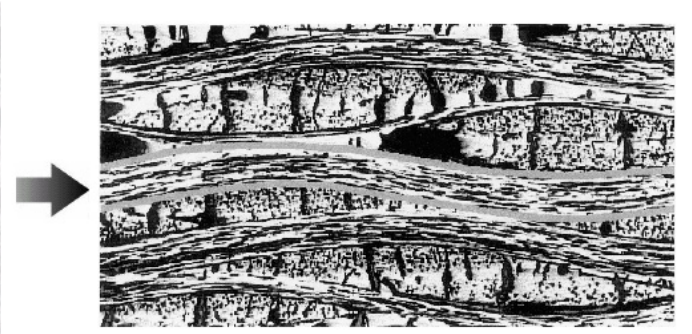

(b)

Figure 2: a) Plane view of textile composite, b) Binary image of transverse section, c) Mesoscopic SEPUC.

ing the material statistics of the real microstructure with those corresponding to simplified periodic unit cell in the framework of a certain optimization problem then allows for deriving the desired geometrical parameters needed in the construction of individual SEPUCs. Knowing the periodic unit cell then opens the way for the derivation of effective elastic properties using the well known elements of first order homogenization procedure briefly outlined in Section 3. 


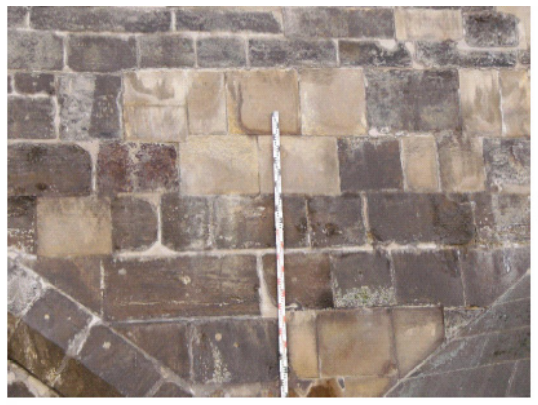

(a)

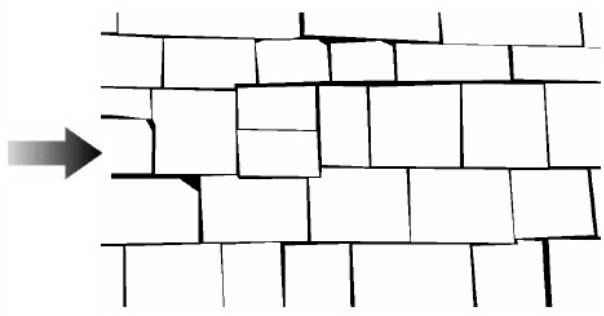

(b)

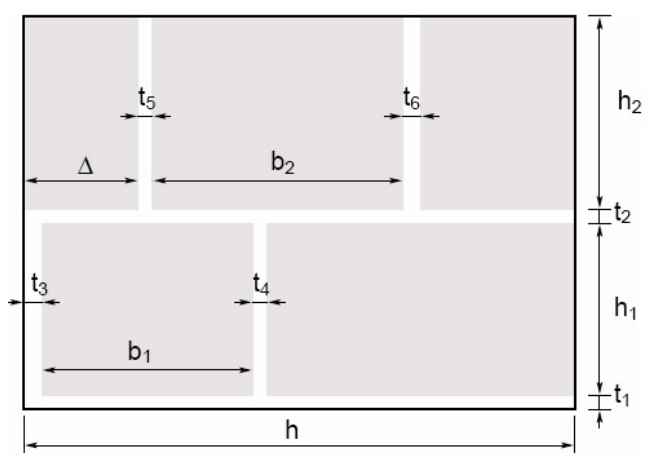

(c)

Figure 3: a) Parapet wall, b) Binary image, c) Mesoscopic SEPUC.

\section{Definition of a periodic unit cell}

The crucial step in the definition of optimal periodic unit cell relies on the choice of proper description of heterogeneous materials with random or imperfect structure. In the present work, we restrict our attention to two specific descriptors: one- and two-point probability functions $S_{r}$ and $S_{r s}$; see, e.g., [5] for more details.

To that end, consider a binary heterogenous material formed by phases $r$ and $s$ and denote the characteristic function of the domain occupied by the $r$-th phase $\chi_{r}$. (When referring to textiles the symbols $r$ and $s$ may represent the fiber (fiber tow) and matrix phases, while for masonry the two symbols are essentially reserved for brick (stone) and mortar phases.) Then, the one-point probability function gives the probability that a point $\mathbf{x}$ will be found in a given phase $r$ and the two-point probability function $S_{r s}$ stands for the probability that the points $\mathbf{x}$ and $\mathbf{y}$ will be located simultaneously in phases $r$ and $s$, respectively:

$$
S_{r}(\mathbf{x})=P\left(\chi_{r}(\mathbf{x})=1\right), S_{r s}(\mathbf{x}, \mathbf{y})=P\left(\chi_{r}(\mathbf{x}) \chi_{s}(\mathbf{y})=1\right) .
$$

For the case of statistically homogeneous and ergodic media, information contained in the one-point probability function reduces to the volume fraction of 
a given phase $\left(S_{r}=c_{r}\right)$. In addition, the two-point probability function then depends on $(\mathbf{x}-\mathbf{y})$ only and can be obtained from the relation

$$
|\Omega| S_{r s}=F^{-1}\left(\widetilde{\chi_{r}} \cdot \overline{\widetilde{\chi_{s}}}\right)
$$

where $|\Omega|$ is the area of the analyzed domain, $\sim$ and $F^{-1}(\cdot)$ stand for the direct and inverse Fourier transform and - denotes the complex conjugate. For discretized microstructures, Eq. (2) can be rapidly evaluated by the fast Fourier transform even for high-resolution bitmaps. In the present study, the bitmaps of Figs. 1(b)3(b) were employed.

Once the original structure has been characterized by an appropriate statistical descriptor, we can proceed with the definition of the idealized unit cell. A particular parameterization considered in this work appears in Figs. 1(c)-3(c). In case of masonry structures (for textile composites we refer the interested reader to [2, 4], Fig. 3(c), the unit cell is fully determined once the width of the unit cell, heights of each layer of bricks and thicknesses of individual joints are specified. In particular, the geometry of the chosen unit cell is determined by twelve parameters. The statistically optimal values of these parameters then follow from minimization of the least square error

$$
E=\sum_{i} \sum_{j}\left(S_{r s}^{0}(i, j)-S_{r s}(i, j)\right)^{2},
$$

where $S_{r s}^{0}$ is the two-point probability function related to the original microstructure while $S_{r s}$ stands for the two-point probability function of the idealized unit cell. It can be shown that the objective function $E$ is non-convex, multi-modal and discontinuous due to the effect of limited bitmap resolution. Based on our previous works, a stochastic global optimization algorithm based on combination of real-valued genetic algorithms and the simulated annealing method, see [6], is employed to solve this optimization problem. This approach was successful in delivering the desired periodic unit cells for all material systems considered herein.

\section{First order homogenization}

Consider a heterogenous periodic unit cell $Y$ subjected to a uniform macroscopic (mesoscopic) strain $\boldsymbol{E}$. In view of the periodicity of the unit cell, the strain and displacement fields in the PUC admit the following decomposition

$$
\mathbf{u}(\mathbf{x})=\boldsymbol{E} \cdot \mathbf{x}+\mathbf{u}^{*}(\mathbf{x}), \quad \varepsilon(\mathbf{x})=\boldsymbol{E}+\varepsilon^{*}\left(\mathbf{u}^{*}(\mathbf{x})\right) .
$$

The first term on the right hand side of Eq. (4) corresponds to a displacement field in an effective homogeneous medium which has the same overall response as the composite aggregate. The fluctuating $Y$-periodic displacement $\mathbf{u}^{*}$ and corresponding strain $\varepsilon^{*}$ enter Eqs. (4) as a consequence of the presence of heterogeneities; see, e.g., [7] and references therein. Note that the periodicity of $\mathbf{u}^{*}$ further implies 


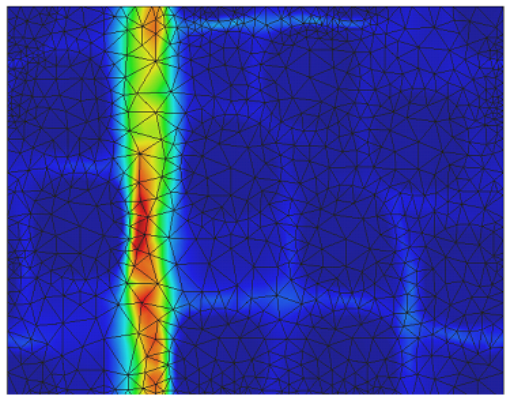

(a)

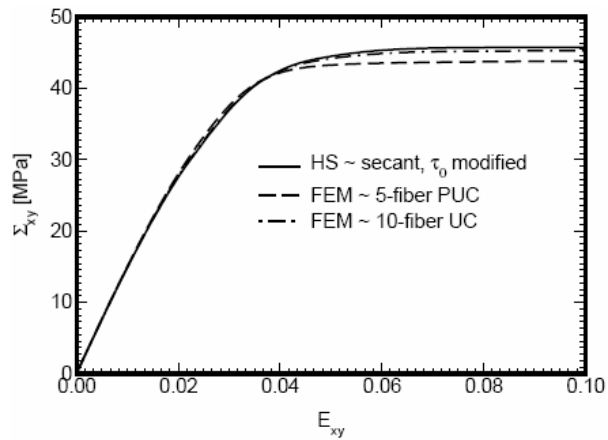

(b)

Figure 4: (a) Localized equivalent strain, (b) Mesoscopic response for 5-fiber and 10-fiber PUCs.

that the average of $\varepsilon^{*}$ in the unit cell vanishes. The local stress fields $\sigma$ in the PUC are constrained by constitutive equations and equilibrium conditions

$$
\boldsymbol{\sigma}(\mathbf{x})=\mathbf{L}(\mathbf{x}): \varepsilon(\mathbf{x}), \quad \operatorname{div} \boldsymbol{\sigma}(\mathbf{x})=\mathbf{0},
$$

respectively. Note that symbol $\mathbf{L}$ stands for the (position-dependent) fourth-order symmetric stiffness tensor. Combining Eqs. (5) and (4) allows us to determine the distribution of fluctuating displacement $\mathbf{u}^{*}$ within the unit cell as a function of $\boldsymbol{E}$ and, subsequently, to evaluate the average stress in the PUC as

$$
\boldsymbol{\Sigma}=\langle\boldsymbol{\sigma}\rangle=\frac{1}{|Y|} \int_{Y} \boldsymbol{\sigma}\left(\mathbf{u}^{*}(\boldsymbol{E})\right) \mathrm{d} Y, \text { in particular, } \boldsymbol{\Sigma}=\mathbf{L}^{\text {hom }}: \boldsymbol{E},
$$

where $\mathbf{L}^{\text {hom }}$ is the homogenized stiffness tensor characterizing the equivalent elastic homogeneous medium.

\subsection{Mesoscopic response of polymer matrix based fibrous composites}

Application of Eq. (6) to the derivation of mesoscopic response of graphite fiber tow impregnated by the polymer matrix is shown in Fig. 4. In this particular example an influence of the number of fibers within the PUC on the mesoscopic response was explored. While the elastic behavior is essentially independent of the number of fibers assumed for the PUC (note that even the model with hexagonal arrangement of fibers gives the same elastic response) the nonlinear behavior suggests possible dependency. This may be attributed to significant non-homogeneous distribution of local fields manifested, e.g., by highly localized zones of equivalent local strain, Fig. 4(a), when loading this system beyond the elastic limit. It should be mentioned that in this particular example the response of the matrix phase was assumed to be well described by the generalized nonlinear viscoelastic Leonov model [8]. 


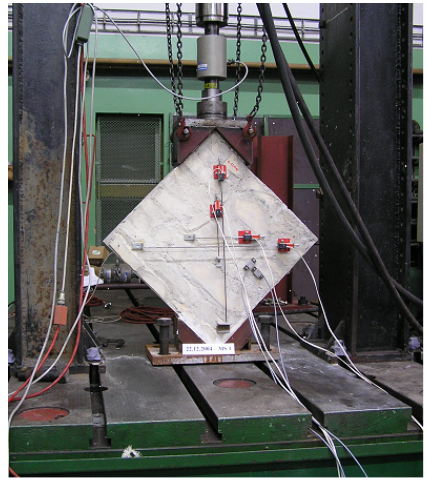

(a)

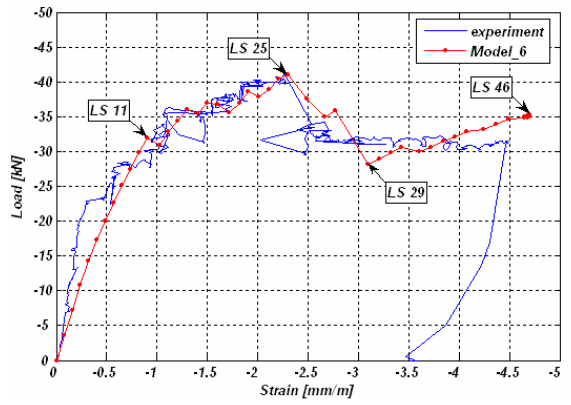

(c)

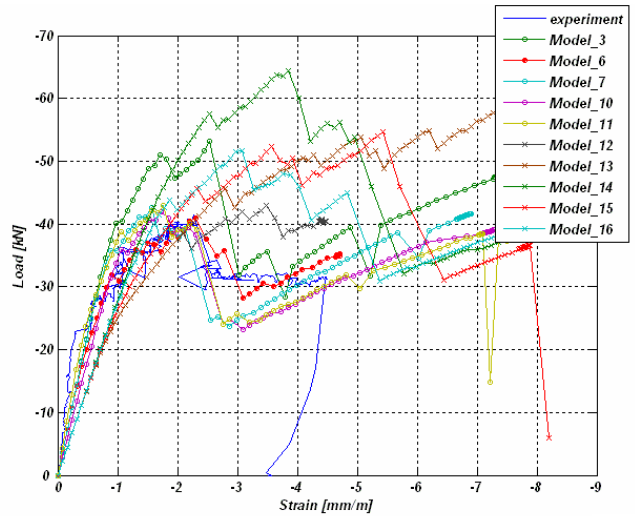

(b)

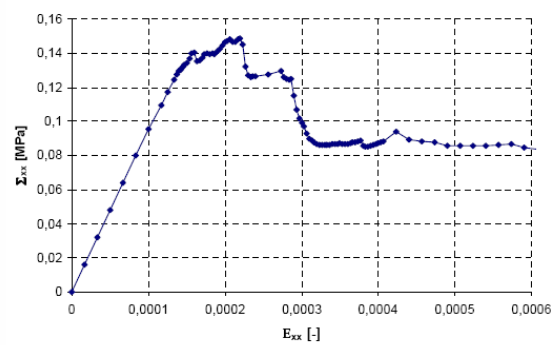

(d)

Figure 5: (a) Experimental setup, (b) Simulations for various material parameters of interface elements, (c) Experimental vs. numerical response, (d) Mesoscopic response.

\subsection{Evaluation of effective fracture energy of masonry}

The second example demonstrates the use of homogenization theory to the derivation of macroscopic fracture energy as a material property needed in the large scale analysis of historical structures such as bridges, where detailed modeling of individual phases (stones and mortar) is essentially impossible. This property is found as the area under the macroscopic stress-strain curve, Fig. 5(d), multiplied by the PUC area and divided by the total crack length as suggested in [9]. In analogy with the smeared crack model assumed for individual phases, see [10] for more details, it can be shown that for the unit cell, Fig. 3(c), loaded by macroscopically uniform stress or strain in the direction of one of the coordinate axis, say width $h$, (all cracks are then assumed to be perpendicular to the loading direction with the length approaching the other unit cell dimension $h_{1}+h_{2}+t_{1}+t_{2}$ ) the macroscopic fracture energy receives the value equal to the area under the macroscopic stress-strain curve multiplied by the crack width $h$. In such a particular case the 
two definitions are not only identical, but yet confirm applicability of the homogenization theory even for quasi-brittle materials.

To arrive at reliable macroscopic response, however, may prove to be rather complicated. General assumption of perfect and strong bond between individual phases is usually not acceptable due to weakening effect of air bubbles in the stone phase in the vicinity of stone-mortar interface. In our particular case, this zone was represented by interface elements with the behavior governed by the Mohr-Coulomb failure criterion. The model parameters such as cohesion and angel of internal friction were derived by matching numerical simulations, Figs. 5(b)(c), with the results derived experimentally, Fig. 5(a). Although more sophisticated techniques based on inverse approach are available in the literature, a simple trial and error method was exercised here to fit individual parameters of the assumed constitutive model. In particular, a series of possible solutions displayed in Fig. 5(b) was derived based on randomly generated values of the cohesion and angel of internal friction. Material parameters corresponding to the "best" solution, see Fig. 5(c), were then applied in the unit cell analysis to derive the required macroscopic response to a sufficient degree of accuracy.

\section{Conclusion}

A rather general approach to the analysis of heterogeneous materials with either random or imperfect microstructures was reviewed. The basic scheme assumes formulation of a certain periodic unit cell statistically equivalent (up to two-point probability function) to real material systems. It is expected that the periodic unit cell being statistically similar (from the geometrical point of view) to real systems will also provide similar (at best the same) mechanical response. The robustness of this approach has been demonstrated through applications to rather different material systems varying from plane weave textiles over to natural stone masonry. A special attention was further devoted to the derivation of effective fracture energy of masonry systems to support applicability of homogenization techniques also to quasi-brittle materials.

\section{Acknowledgment}

The financial support provided by GAČR Grant No. 103/04/1321 and CEZ MSM 6840770003 is gratefully acknowledged.

\section{References}

[1] Dvorak, G.J., Bahei-El-Din, Y.A. \& Wafa, A., Implementation of the transformation field analysis for inelastic composite-materials. Computational Mechanics, 14(3), pp. 201-228, 1994.

[2] Zeman, J. \& Šejnoha, M., Numerical evaluation of effective properties of graphite fiber tow impregnated by polymer matrix. Journal of the Mechanics and Physics of Solids, 49(1), pp. 69-90, 2001. 
[3] Šejnoha, M. \& Zeman, J., Overall viscoelastic response of random fibrous composites with statistically quasi uniform distribution of reinforcements. Computer Methods in Applied Mechanics and Engineering, 191(44), pp. 5027-5044, 2002.

[4] Zeman, J. \& Šejnoha, M., Homogenization of balanced plain weave composites with imperfect microstructure: Part i - theoretical formulation. International Journal for Solids and Structures, 41(22-23), pp. 6549-6571, 2004.

[5] Torquato, S., Random heterogeneous materials: Microstructure and macroscopic properties. Springer-Verlag, 2002.

[6] Matouš, K., Lepš, M., Zeman, J. \& Šejnoha, M., Applying genetic algorithms to selected topics commonly encountered in engineering practice. Computer Methods in Applied Mechanics and Engineering, 190(13-14), pp. 16291650, 2000.

[7] Michel, J.C., Moulinec, H. \& Suquet, P., Effective properties of composite materials with periodic microstructure: A computational approach. Computer Methods in Applied Mechanics and Engineering, 172, pp. 109-143, 1999.

[8] Leonov, A.I., Non-equilibrium thermodynamics and rheology of viscoelastic polymer media. Rheol Acta, 15, pp. 85-98, 1976.

[9] RILEM TC50 FMC, Determination of the fracture energy of mortar and concrete by means of three-point bend tests on notched beams. Materials and Structures, 18, pp. 287-290, 1985. Endorsed May 1993.

[10] Červenka, V., Jendele, L. \& Červenka, J., ATENA Program DocumentationPart I : Theory. Červenka Consulting Company, 2002. 